Research Article

\title{
On the Analytical Solutions of the Forced Damping Duffing Equation in the Form of Weierstrass Elliptic Function and its Applications
}

\author{
S. A. El-Tantawy $\mathbb{D}^{1,2}$ Alvaro H. Salas $\mathbb{D}^{1,}{ }^{3}$ and M. R. Alharthi $\mathbb{D}^{4}$ \\ ${ }^{1}$ Department of Physics, Faculty of Science, Port Said University, Port Said 42521, Egypt \\ ${ }^{2}$ Research Center for Physics (RCP), Department of Physics, Faculty of Science and Arts, Al-Baha University, \\ Al-Mikhwah, Saudi Arabia \\ ${ }^{3}$ Department of Mathematics, Universidad Nacional de Colombia, \\ Universidad Nacional de Colombia-Nubia Campus Department of Mathematics and Statistics FIZMAKO Research Group, \\ Bogota, Colombia \\ ${ }^{4}$ Department of Mathematics and Statistics, College of Science, Taif University, P.O.Box 11099, Taif 21944, Saudi Arabia
}

Correspondence should be addressed to S. A. El-Tantawy; samireltantawy@yahoo.com

Received 1 December 2020; Revised 30 January 2021; Accepted 3 February 2021; Published 16 February 2021

Academic Editor: Sundarapandian Vaidyanathan

Copyright (c) 2021 S. A. El-Tantawy et al. This is an open access article distributed under the Creative Commons Attribution License, which permits unrestricted use, distribution, and reproduction in any medium, provided the original work is properly cited.

\begin{abstract}
In this study, a novel analytical solution to the integrable undamping Duffing equation with constant forced term is obtained. Also, a new approximate analytical (semianalytical) solution for the nonintegrable linear damping Duffing oscillator with constant forced term is reported. The analytical solution is given in terms of the Weierstrass elliptic function with arbitrary initial conditions. With respect to it, the semianalytical solution is constructed depending on a new ansatz and the exact solution of the standard Duffing equation (in the absence of both damping and forced terms). A comparison between the obtained solutions and the Runge-Kutta fourth-order (RK4) is carried out. Moreover, some complicated oscillator equations such as the constant forced damping pendulum equation, forced damping cubic-quintic Duffing equation, and constant forced damping Helmholtz-Duffing equation are reduced to the forced damping Duffing oscillator, in which its solution is known. As a practical application, the proposed techniques are applied to investigate the characteristics behavior of the signal oscillations arising in the RLC circuit with externally applied voltage.
\end{abstract}

\section{Introduction}

Since the early century until now, Duffing equation [1-3] has been devoted by many authors in order to investigate the nonlinear oscillations in engineering technology fields and in several physical systems including electrical and mechanical with nonlinear restoring force [4]. So far, this equation and its family have remained a good model in studying and explaining many nonlinear structures in the dynamic systems and various branches of sciences [5]. This family is considered an excellent example for the dynamic system that exhibits chaotic behavior. The Duffing equation with a cubic stiffness term was introduced for the first time by Duffing [6] in 1918 for describing the hardening spring effect observed in many mechanical problems. Since then, this equation has become one of the commonest examples in nonlinear oscillation texts and research articles $[4,7]$.

The exact analytic solutions of the standard (unforced and undamping) Duffing equation $\left(\ddot{x}+\alpha x+\beta x^{3}=0\right)$ and the cubic-quintic Duffing equation $\left(\ddot{x}+p x+q x^{3}+r x^{5}=0\right)$ have been obtained by many authors in terms of Jacobian elliptic functions [8-14]. Since most realistic physical systems are subjected to the influence of some frictional forces, these forces must be taken into account in Duffing equation to become unforced damping Duffing equation $(\ddot{x}+2 \gamma \dot{x}+$ $\left.\alpha x+\beta x^{3}=0\right)[15-19]$. The unforced undamping Duffing 
equation has been solved numerically using the differential transform method, and the author found that both numerical and exact analytic solutions coincide with each other [13]. Also, the analytical solution of the unforced damping Duffing equation in terms of Jacobian elliptic functions has been derived by Johannessen [17, 18]. Moreover, Johannessen $[17,18]$ made a comparison between the analytical solution and the approximate numerical solution using RK4, and he found that the two solutions were largely identical.

In some physical and engineering systems, the system can be excited by an external force, and this force may be constant or a function of the time. In this case, for modeling the oscillations in these systems, the excited force must be taken into consideration in the Duffing equation which we finally obtain as the forced damping Duffing equation $\left(\ddot{x}+2 \gamma \dot{x}+\alpha x+\beta x^{3}=F\right)$. Some authors studied the Duffing equation solutions with (out) both damping term and driving/external force [8, 20-24]. For instance, the exact solutions of both undamped Duffing equation and forced undamped Duffing equation have been derived in the form of Jacobian elliptic functions by Hsu [8]. Furthermore, the Jacobian elliptic functions have been devoted for getting the approximate solution of the forced damped Duffing equation [20]. This new equation of motion has many applications in electrical and mechanical systems [21, 22] as well as in different branches of science such as studying the oscillations in plasma physics. For example, the forced damping Duffing equation with constant force can be used for investigating the nonlinear oscillations in RLC circuits if the circuit has DC battery. Moreover, the forced damping Duffing equation can be used for modeling the damping oscillations in different plasma models. For instance, for any plasma system having a critical value for its related parameters, we can reduce the fluid equations of the plasma species to a modified Korteweg-de Vries-Burgers (mKdVB) equation $\left(\partial_{t} \phi+A_{p} \phi^{2} \partial_{x} \phi+B_{p} \partial_{x}^{3} \phi-C_{p} \partial_{x}^{2} \phi\right)$ using the reductive perturbation technique (RPT). After that the $\mathrm{mKdVB}$ can be transformed to the forced damping Duffing equation by means of a traveling wave transformation for studying the damping oscillations in the plasma system. It should be noted here that the last term in the $\mathrm{mKdV}$ equation appeared as a result of taking the kinematic viscosity of some plasma species into account. Motivated by the mentioned investigations, we restrict our attention for studying and solving the forced damping Duffing equation,

$$
\left\{\ddot{x}+2 \gamma \dot{x}+\alpha x+\beta x^{3}=F, x(0)=x_{0} \& x^{\prime}(0)=\dot{x}_{0},\right.
$$

and some related equations using some new approaches. Thus, our study will be divided into two main goals/parts. First, we will solve the forced damping Duffing equation in the absence of the friction force $(\gamma=0)$ in order to get an exact solution in terms of Weierstrass elliptic function. In the second part, a new ansatz will be utilized with the help of some exact solution of standard Duffing equation $(\gamma=F=0)$ in order to find an approximate analytic solution for the initial value problem (1).

\section{Methodology}

In the beginning, we dissect the i.v.p (1) into two cases and then solve them. In the first case, the undamping Duffing equation with perturbation/excitation force, i.e., $\gamma=0$ and $F \neq 0$, is considered. This case has several applications in plasma physics, oceans, mechanical fluid, and electronic circuits in the absence viscosity and friction forces. In the second case, the linear damping Duffing equation with perturbation force (sometimes is called the constant forced damping Duffing equation), i.e., $\gamma \neq 0$ and $F \neq 0$, is considered. Also, this case has various applications in plasma physics if the kinematic viscosity of the plasma species is taken into account or if the dust fluctuations are taken into consideration.

2.1. Case I: An Exact Analytic Solution for the Forced Undamping Duffing Equation. If the damping term in the i.v.p (1) is neglected $(\gamma=0)$, then the i.v.p (1) reduces to the following integrable i.v.p.

$$
\left\{\ddot{x}+p x+q x^{3}=F, x(0)=x_{0} \text { and } x^{\prime}(0)=\dot{x}_{0} .\right.
$$

Let us assume that the following solution satisfies the i.v.p. (2)

$$
\eta(t)=\lambda+\frac{\mu}{1+\rho \gamma},
$$

where $\wp \equiv \wp\left(t+t_{0} ; g_{2}, g_{3}\right)$ is the Weierstrass elliptic function and satisfies the following condition:

$$
\wp^{\prime 2}=4 \wp^{3}-g_{2} \wp-g_{3}
$$

By inserting solution (3) into equation (2) and taking relation (4) into consideration, we get

$$
\sum_{j=0}^{3} W_{j} \wp^{j}=0
$$

with

$$
\begin{aligned}
W_{0}= & -2 F-4 g_{3} \mu \rho^{2}+g_{2} \mu \rho+2 p \lambda+2 p \mu \\
& +2 q \lambda^{3}+6 q \lambda^{2} \mu+6 q \lambda \mu^{2}+2 q \mu^{3} \\
W_{1}= & -\rho\left(\begin{array}{c}
6 F+3 g_{2} \mu \rho-6 p \lambda-4 p \mu \\
-6 q \lambda^{3}-12 q \lambda^{2} \mu-6 q \lambda \mu^{2}
\end{array}\right), \\
W_{2}= & 2 \rho\left(\begin{array}{c}
-3 F \rho+3 p \lambda \rho+p \mu \rho \\
+3 q \lambda^{3} \rho+3 q \lambda^{2} \mu \rho-6 \mu
\end{array}\right), \\
W_{3}= & 2 \rho^{2}\left(-F \rho+p \lambda \rho+q \lambda^{3} \rho+2 \mu\right) .
\end{aligned}
$$

By equating the coefficients $W_{j}$ to zero, a system of algebraic equations is obtained, and by solving this system, the values of $\mu, \rho, g_{2}$, and $g_{3}$ are obtained as follows: 


$$
\begin{aligned}
\mu & =\frac{6\left(F-\lambda\left(p+q \lambda^{2}\right)\right)}{p+3 q \lambda^{2}}, \\
\rho & =\frac{12}{p+3 q \lambda^{2}}, \\
g_{2} & =\frac{1}{12}\left(-3 q \lambda\left(q \lambda^{3}-4 F\right)+p^{2}-6 p q \lambda^{2}\right), \\
g_{3} & =\frac{1}{216}\left(27 F^{2} q+9 p q \lambda\left(q \lambda^{3}-4 F\right)+p^{3}+18 p^{2} q \lambda^{2}\right) .
\end{aligned}
$$
get

By applying the initial conditions given in i.v.p (2), we

$$
t_{0}=\wp^{-1}\left(\frac{\lambda+\mu-x_{0}}{\rho\left(x_{0}-\lambda\right)} ; g_{2}, g_{3}\right)
$$

The value of $\lambda$ is a solution to the following quartic equation:

$$
q \lambda^{4}+2 p \lambda^{2}-4 F \lambda+\left(4 F x_{0}-2 p x_{0}^{2}-q x_{0}^{4}-2 \dot{x}_{0}^{2}\right)=0 .
$$

Note that the solution (3) is periodic with period

$$
T=2 \int_{r}^{\infty} \frac{\mathrm{d} x}{\sqrt{4 x^{3}-g_{2} x-g_{3}}},
$$

where $r$ is the greatest real root of the cubic equation: $4 x^{3}-g_{2} x-g_{3}=0$.

In equation (9), let us discuss the value of the following number:

$$
\Delta=-\frac{1}{q}\left(4 F x_{0}-2 p x_{0}^{2}-q x_{0}^{4}-2 \dot{x}_{0}^{2}\right)
$$

which is called the discriminant of the i.v.p. (2). If equation (9) has at least one real root, then all parameters that are given in equation (7) become real for real $\lambda$. On the other side, if all roots of equation (9) are complex, then $\Delta<0$. Indeed, let $r_{1}, \ldots, r_{4}$ be the roots of equation (9), and then

$$
r_{1} r_{2} r_{3} r_{4}=\frac{1}{q}\left(4 F x_{0}-2 p x_{0}^{2}-q x_{0}^{4}-2 \dot{x}_{0}^{2}\right)=-\Delta>0,
$$

so that $\Delta<0$, and in this case, we cannot obtain real values for the parameters given in equation (7). In order to obtain a solution to the i.v.p. (2) with real values to parameters $\left(\mu, \rho, g_{2}, g_{3}\right)$, we make the following substitution:

$$
x(t)=s-\frac{2 s}{1+v(t)},
$$

where $s \neq 0$, and $v \equiv v(t)$ is a solution to the following Duffing equation:

$$
\ddot{v}+M v+N v^{3}=F_{0} .
$$

Taking into account the initial conditions given in the i.v.p. (2), we obtain

$$
\begin{aligned}
v(0) & =\frac{2 s \dot{x}_{0}}{\left(s-x_{0}\right)^{2}}, \\
v^{\prime}(0) & =\frac{s+x_{0}}{s-x_{0}}
\end{aligned}
$$

On the other hand, the first integration of equation (14) gives

$$
v^{\prime}(t)^{2}=C+2 F_{0} v(t)-M v(t)^{2}-\frac{1}{2} N v(t)^{4},
$$

and by applying initial condition given in the i.v.p. (2), we get

$$
C=\frac{1}{2\left(s-x_{0}\right)^{4}}\left[\begin{array}{c}
-4 F_{0}\left(s-x_{0}\right)^{3}\left(s+x_{0}\right)+2 M\left(s^{2}-x_{0}^{2}\right)^{2} \\
+N\left(s+x_{0}\right)^{4}+8 s^{2} \dot{x}_{0}^{2}
\end{array}\right],
$$

with

$$
\begin{aligned}
& N=\frac{-F+p s+q s^{3}}{2 s}, \\
& M=\frac{3 F-p s+3 q s^{3}}{2 s}, \\
& F_{0}=-\frac{F}{s} .
\end{aligned}
$$

Inserting equations (14)-(17) into the first equation (here, $\mathbb{R} \equiv \ddot{x}+p x+q x^{3}-F$ ) of i.v.p (2), we have

$$
\mathbb{R}=-\frac{8 q s^{3}}{(1+v(t))^{3}\left(s-x_{0}\right)^{4}}\left(s^{4}+\frac{2 F s}{q}+\Delta\right) .
$$

Observe that the quartic equation,

$$
s^{4}+\frac{2 F}{q} s+\Delta=0
$$

has at least one real root, since $\Delta<0$. Thus, for $\Delta<0$, the solution of the i.v.p. (14) and (15) expresses the solution of the i.v.p. (2), where the parameters $\left(M, N, F_{0}\right)$ are given in equation (18).

Example 1. The solution of the following i.v.p. according to the relation (3)

$$
\left\{\ddot{x}+x+x^{3}=1, x(0)=1 \text { and } x^{\prime}(0)=1,\right.
$$


reads

$$
x(t)=1.29637-\frac{2.45792}{1+1.98619 \wp(0.521719-t ;-0.166667,0.171296)} .
$$

Figure 1 illustrates the comparison between the solution (22) and the RK4 numerical solution. It is known that RK4 numerical solution to the ordinary differential equations is the best so far. Thus, our solution is compared to it, and it is found that the obtained results are completely compatible with each other. Moreover, the periodicity of solution (22) is given by $T=4.24727$.

\subsection{Case II: An Approximate Analytic Solution for the Forced} Damping Duffing Equation. It is known that the forced damping Duffing equation $\left(\mathbb{R}=\ddot{x}+2 \gamma \dot{x}+\alpha x+\beta x^{3}-F\right)$ is not integrable unless $F=0$ and $\alpha=(8 / 9) \gamma^{2}$. Thus, in this subsection, we seek an approximate analytic solution to (1) in the form

$$
x(t)=d+\exp (-\gamma t) \eta(t)
$$

where $\eta \equiv \eta(t)$ is a solution to standard Duffing equation $\left(\ddot{x}+\alpha x+\beta x^{3}=0\right)$, and $d$ is some constant to be determined later.

Substituting solution (23) into the forced damping Duffing equation $\left(\mathbb{R} \equiv \ddot{x}+2 \gamma \dot{x}+\alpha x+\beta x^{3}-F=0\right)$, we have

$$
\begin{aligned}
\mathbb{R}= & \left(\beta d^{3}+\alpha d-F\right) \\
& +\left(3 d^{2} \beta e^{-t \gamma}+\alpha e^{-t \gamma}-\gamma^{2} e^{-t \gamma}\right) \eta(t) \\
& +3 d \beta e^{-2 t \gamma} \eta(t)^{2}+\beta e^{-3 t \gamma} \eta(t)^{3}+e^{-t \gamma} \eta^{\prime \prime}(t)
\end{aligned}
$$

For small $\gamma \ll 1$ and not too large $t, e^{\gamma t} \approx 1$, so that equation (24) reduces to

$$
\begin{aligned}
\mathbb{R} \approx & \eta^{\prime \prime}(t)+\left(3 d^{2} \beta+\alpha-\gamma^{2}\right) \eta(t) \\
& +3 d \beta \eta(t)^{2}+\beta \eta(t)^{3} \\
& +\left(\beta d^{3}+\alpha d-F\right) .
\end{aligned}
$$

The value of $d$ could be obtained by equating the last term in equation (25) to zero:

$$
\beta d^{3}+\alpha d-F=0
$$

Accordingly, the following new i.v.p. needs to solve.

$$
\left\{\begin{array}{l}
\eta^{\prime \prime}(t)+\left(3 d^{2} \beta+\alpha-\gamma^{2}\right) \eta(t) \\
+3 d \beta \eta(t)^{2}+\beta \eta(t)^{3}=0, \\
\eta(0)=\dot{x}_{0}+\gamma\left(x_{0}-d\right), \\
\eta^{\prime}(0)=x_{0}-d .
\end{array}\right.
$$

Inserting the hypothesis $\eta(t)=z(t)-d$ into the i.v.p. (27), we get

$$
\left\{\begin{array}{l}
z^{\prime \prime}(t)+\widetilde{\alpha} z(t)+\beta z(t)^{3}=\widetilde{F} \\
z(0)=\dot{x}_{0}+\gamma\left(x_{0}-d\right) \& z^{\prime}(0)=x_{0}
\end{array}\right.
$$

where $\widetilde{\alpha}=\left(\alpha-\gamma^{2}\right)$ and $\widetilde{F}=\left(F-\mathrm{d} \gamma^{2}\right)$. Observe that the i.v.p. (28) refers to the constant forced undamping Duffing equation. From previous section, we already know how to solve this problem. Then, an approximate analytical solution to the constant forced damping Duffing equation (1) could be given by

$$
x(t)=d+\exp (-\gamma t)(z(t)-d)
$$

where the number $d$ is a solution to the cubic equation (26), and $z=z(t)$ is the exact solution of the i.v.p. (28). Note that for $\gamma>0$, the value of parameter $d$ must be chosen according: $\lim _{t \rightarrow \infty} x(t)=d$.

Example 2. Let us analyze the i.v.p. (1) using different values for $(\gamma, \alpha, \beta, F)$. The solutions of the i.v.p. (1) according to the relation (23) and $(\gamma, \alpha, \beta, F)=(0.1,2.2,1,0.01)$ and $(\gamma, \alpha, \beta, F)=(0.1,2.2,1,0)$ are, respectively, given by

$$
\begin{aligned}
& x(t)=0.00454541+e^{-0.1 t}\left(0.13229-\frac{0.780732}{1+5.34242 \wp(t+3.15199 ; 0.380447,0.0556578)}\right), \\
& x(t)=e^{-0.1 t}\left(0.134868-\frac{0.796091}{1+5.34624 \wp(t+3.17447 ; 0.379675,0.0559271)}\right) .
\end{aligned}
$$

Figures 2(a) and 2(b), respectively, demonstrate the comparison between the approximate analytical solutions
(30) and (31) and the RK4 approximate solution. Also, the maximum distance error $\left(L_{D}\right)$ with respect to RK4 is 


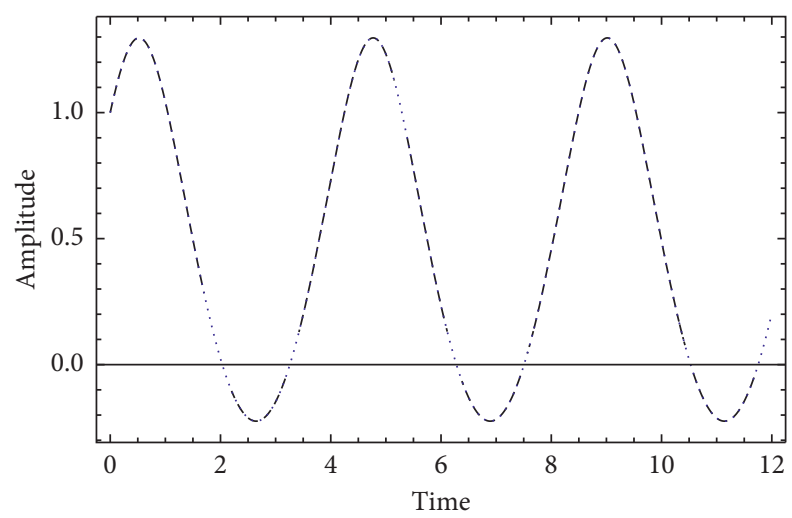

FIgURE 1: The comparison between the analytical solution (20) (dotted curve) and the RK4 numerical solution (dashed curve) for the forced undamping Duffing equation (21) is investigated for $(\gamma, \alpha, \beta, F)=(0,1,1,1)$.

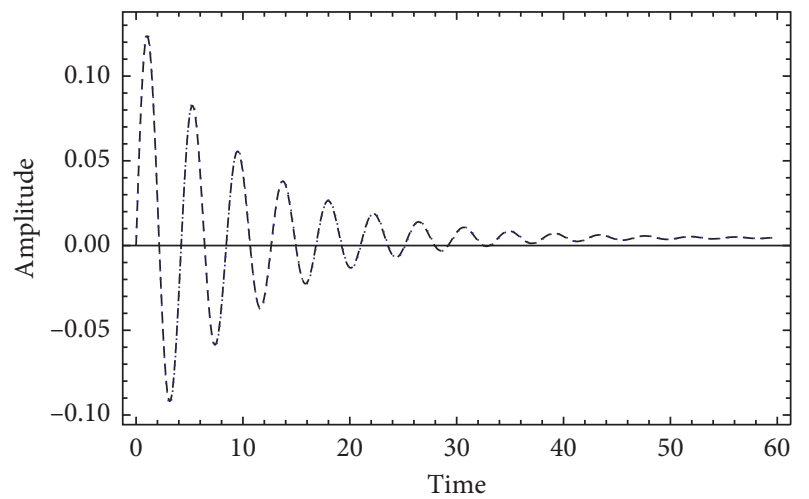

(a)

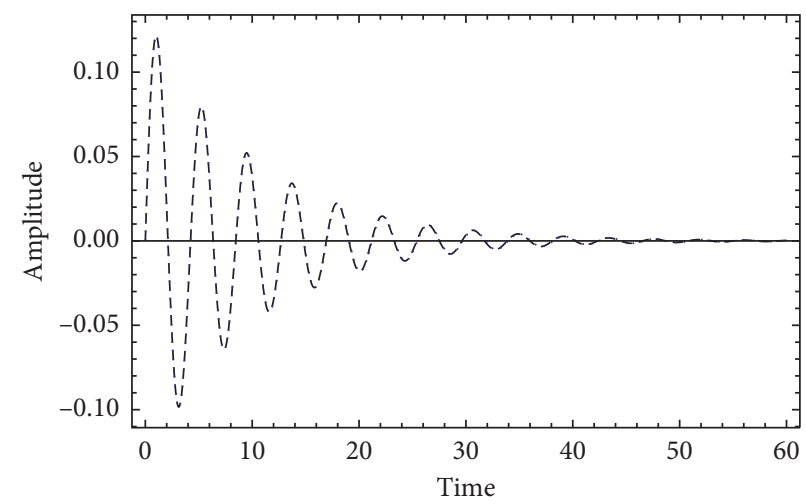

(b)

Figure 2: The comparison between the approximate analytic solutions (30) and (31) (dotted curve) and the RK4 numerical solution (dashed curve) for the forced damping Duffing equation (22) is carried out different values for $(\gamma, \alpha, \beta, F)$.

estimated for different values of the parameters $(\gamma, \alpha, \beta, F)$ as shown in Table 1.

It is noticed from Figure 2 and the values of distance error $\left(L_{D}\right)$ given in Table 1 that the semianalytical solution (23) gives good results as compared to the approximate numerical solution using RK4. Also, it is seen that the distance error decreases with the enhancement of the coefficient of the damping term $(\gamma)$, while the forced term $(F)$ has an opposite effect on the distance error, i.e., increasing $F$ leads the enhancement of the distance error $L_{D}$. Moreover, our solution gives good results for $\alpha>\beta$. Also, it is noted that the distance error $\left(L_{D}\right)$ decreases as the difference between $\alpha$ and $\beta$ is large.

\section{Applications}

Here, we try to find the link between the constant forced damping Duffing equation and some physical and engineering problems related to this equation in order to investigate the nonlinear oscillations in various fields of
TABLE 1: The maximum distance error $\left(L_{D}\right)$ is estimated for $(\alpha, \beta)=(2.2,1)$ and different values of $(\gamma, F)$.

\begin{tabular}{lcc}
\hline$(\gamma, F)$ & Time range & $L_{D}$ \\
\hline$(0,0)$ & $0 \leq t \leq 60$ & $2.45967 \times 10^{-6}$ \\
$(0.01,0)$ & $0 \leq t \leq 60$ & 0.00829489 \\
$(0.1,0)$ & $0 \leq t \leq 60$ & 0.00142482 \\
$(1,0)$ & $0 \leq t \leq 60$ & 0.000434655 \\
$(0.1,0.01)$ & $0 \leq t \leq 60$ & 0.0021093 \\
$(0.1,0.1)$ & $0 \leq t \leq 60$ & 0.0272933 \\
\hline
\end{tabular}

physics and engineering such as the oscillations in RLC circuits and plasma physics.

3.1. Forced Damping Pendulum Equation. The most popular law of motion in mechanics is $\mathrm{F}=m a$, where $\mathrm{F}$ is the force, $m$ is the particle mass, and $a$ is the particle acceleration. Thus, we have a pendulum with length $l$ and with a ball of mass $m$ 
moving in a constant gravitational field $g$ and under friction proportional to the particle velocity and external (un)periodic force. Accordingly, the dimensionless differential equation that can describe the pendulum motion is given by

$$
\left\{\begin{array}{l}
\ddot{\theta}+2 \gamma \dot{\theta}+\omega^{2} \sin \theta=F, \\
\theta(0)=\theta_{0} \& \theta^{\prime}(0)=\dot{\theta}_{0} .
\end{array}\right.
$$

Note that

$$
\begin{aligned}
& \min _{a, b} \int_{-(\pi / 2)}^{\pi / 2}\left[\left(a x+b x^{3}\right)-\sin x\right]^{2} \mathrm{~d} x \\
& =\frac{-806400+161280 \pi^{2}-8160 \pi^{4}+\pi^{8}}{2 \pi^{7}} \\
& \approx 0.000024,
\end{aligned}
$$

for

$$
\begin{aligned}
& a=\frac{240\left(21-2 \pi^{2}\right)}{\pi^{5}}, \\
& b=\frac{3360\left(\pi^{2}-10\right)}{\pi^{7}} .
\end{aligned}
$$

Accordingly, and by expanding $\sin \theta=\theta-\left(\theta^{3} / 6\right)$ $+O\left(\theta^{5}\right)$, the i.v.p. (32) could be replaced by the following new i.v.p.

$$
\left\{\begin{array}{l}
\ddot{\theta}+2 \gamma \dot{\theta}+\alpha \theta+\beta \theta^{3}=F, \\
\theta(0)=\theta_{0} \& \theta^{\prime}(0)=\dot{\theta}_{0},
\end{array}\right.
$$

with

$$
\begin{aligned}
& \alpha=\frac{240\left(21-2 \pi^{2}\right) \omega^{2}}{\pi^{5}}, \\
& \beta=\frac{3360\left(\pi^{2}-10\right) \omega^{2}}{\pi^{7}} .
\end{aligned}
$$

Now, the i.v.p. (35) is similar to the i.v.p. (1), which we discussed as its solution in the above sections.

Example 3. The solution of the i.v.p. (32) for $\left(\gamma, \omega, F, \theta_{0}, \dot{\theta}_{0}\right)=(0.1,1,0.1,0,0)$ reads

$$
\theta(t)=0.101286+e^{-0.1 t}\left(-0.102309+\frac{0.612929}{1+12.26 \wp(t+6.22606 ; 0.0798509,0.0041612)}\right) .
$$

The comparison between solution (37) and the numerical solution using RK4 is displayed in Figure 3. Also, the distance error is estimated $\left(L_{D}=0.00560317\right)$. It is observed that the obtained results are completely compatible with each other.

3.2. Forced Damping Cubic-Quintic Duffing Equation. The idea is to replace a cubic-quintic polynomial by an odd parity cubic polynomial. Observe that

$$
\min _{p, q} \int_{-A}^{A}\left[\left(\alpha x+\beta x^{3}+\delta x^{5}\right)-\left(p x+q x^{3}\right)\right]^{2} \mathrm{~d} x=\varepsilon,
$$

for

$$
\begin{aligned}
& p=\alpha-\frac{5 A^{4} \delta}{21}, \\
& q=\frac{10 A^{2} \delta}{9}+\beta, \\
& \varepsilon=\frac{128}{43659} A^{11} \delta^{2} .
\end{aligned}
$$

According to the transformation (38) and (39), the following constant forced damping cubic-quintic Duffing equation

$$
\left\{\ddot{x}+2 \gamma \dot{x}+\alpha x+\beta x^{3}+\delta x^{5}=F, x(0)=x_{0} \& x^{\prime}(0)=\dot{x}_{0},\right.
$$

could be reduced to the following new i.v.p.

$$
\left\{\ddot{x}+2 \gamma \dot{x}+\widetilde{\alpha} x+\widetilde{\beta} x^{3}=F, x(0)=x_{0} \& x^{\prime}(0)=\dot{x}_{0},\right.
$$

with

$$
\begin{aligned}
& \widetilde{\alpha}=\left(\alpha-\frac{5 A^{4} \delta}{21}\right), \\
& \tilde{\beta}=\left(\frac{10 A^{2} \delta}{9}+\beta\right) .
\end{aligned}
$$

Note that the approximation becomes good for small values of $\varepsilon$ which can be achieved for $|\delta| \leq 1$ and $0<A \leq 1$.

Example 4. The solution of the i.v.p. (41) for $\left(\gamma, \widetilde{\alpha}, \beta, \delta, F, x_{0}, \dot{x}_{0}\right)=(0.1,3,0.5,0.1,0.1,0,0)$ reads 


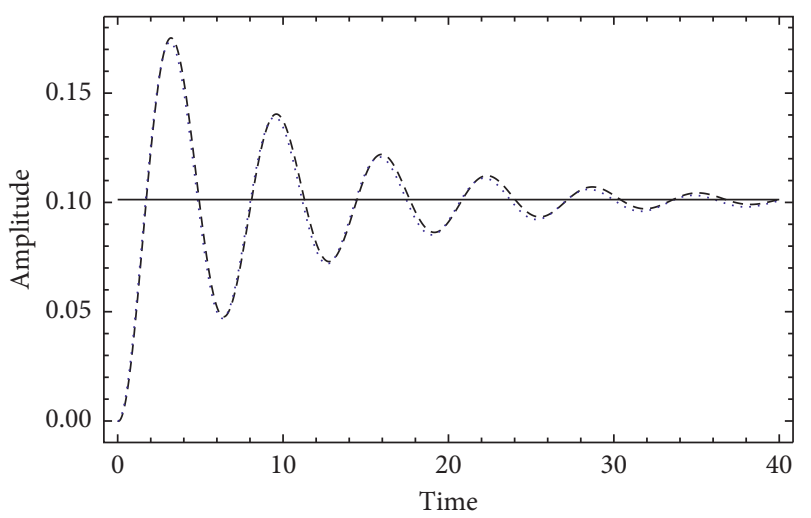

FIgURE 3: The comparison between the solution (37) and the numerical solution using RK4 of the i.v.p. (32) is reported for $\left(\gamma, \omega, F, \theta_{0}, \dot{\theta}_{0}\right)=(0.1,1,0.1,0,0)$.

$$
x(t)=0.0335922+e^{-0.1 t}\left(0.000091584-\frac{0.000549507}{1+4.04044 \wp(t+(1.82295+3.82875 i) ; 0.735051,0.121281)}\right) .
$$

In Figure 4, the comparison between solution (43) and the RK4 numerical solution is introduced. Moreover, the distance error is calculated $\left(L_{D}=0.0304975\right)$.

3.3. Forced Damping Helmholtz-Duffing Equation. Let us consider the following i.v.p.

$$
\left\{\ddot{x}+2 \gamma \dot{x}+p x+q x^{2}+\beta x^{3}=F, x(0)=x_{0} \& x^{\prime}(0)=\dot{x}_{0} .\right.
$$

To convert the i.v.p. (44) to the i.v.p. (1), the following transformation is introduced:

$$
x(t)=z(t)-\frac{q}{3 \beta},
$$

and by substituting this transformation into the i.v.p. (44), we finally get

$$
\left\{\begin{array}{l}
z^{\prime \prime}(t)+2 \gamma z^{\prime}(t)+\widetilde{\alpha} z(t)+\beta z(t)^{3}=\widetilde{\mathrm{F}}, \\
z(0)=x_{0}+\frac{q}{3 \beta} \text { and } z^{\prime}(0)=\dot{x}_{0},
\end{array}\right.
$$

with

$$
\begin{aligned}
& \tilde{\alpha}=\left(p-\frac{q^{2}}{3 r}\right), \\
& \widetilde{\mathrm{F}}=F+\frac{p q}{3 \beta}-\frac{2 q^{3}}{27 \beta^{2}} .
\end{aligned}
$$

This is a constant forced damping Duffing equation with its initial conditions.

3.4. Nonlinear Oscillations in RLC Series Circuits with Applied External Source. In an RLC series circuits consisting of a resistor with resistance $R$ (ohm), an inductor with inductance $L(H)$, and ferroelectric nonlinear capacitor with capacitance $C(F)$ as well as external applied voltage $E(V)$, Kirchhoff's voltage law (KVL) could be written as

$$
L \partial_{t} i^{\prime}(t)+i(t) R+s q+a q^{3}=E
$$

where the relation between the current and charge is given by $i=\partial_{t} q \equiv \dot{q}, i^{\prime} \equiv \partial_{t} i$, the coefficients $(a, s)$ are related to the nonlinear capacitor, and $E$ represents the voltage of the battery which is constant. By reorganizing equation (48), the following constant forced and damped Helmholtz equation could be obtained as

$$
\ddot{q}+2 \gamma \dot{q}+\alpha q+\beta q^{3}=F,
$$

with $\gamma=(R /(2 L)), \alpha=(1 /(L C)), \quad \beta=1 /\left(C q_{0} L\right), \quad$ and $F=E / L$, where $q_{0}=q(t=0)$ is the initial charge value at $t=0, \ddot{q} \equiv \partial_{t}^{2} q$, and $\dot{q} \equiv \partial_{t} q$.

Let us now apply the obtained solution on equation (49) and analyze it numerically using some various values to the coefficients $(\alpha, \beta, \gamma, F)$ according to the RLC series circuit. The solution of the following i.v.p. according to the relation (23) and for $(\alpha, \beta, \gamma, F)=(4,1,0.1,0.2)$ reads

$$
x(t)=0.0499688+e^{-0.1 t}\left(0.130143-\frac{0.770662}{2.93591 \wp(t+2.55163 ; 1.29763,0.318372)+1}\right) .
$$




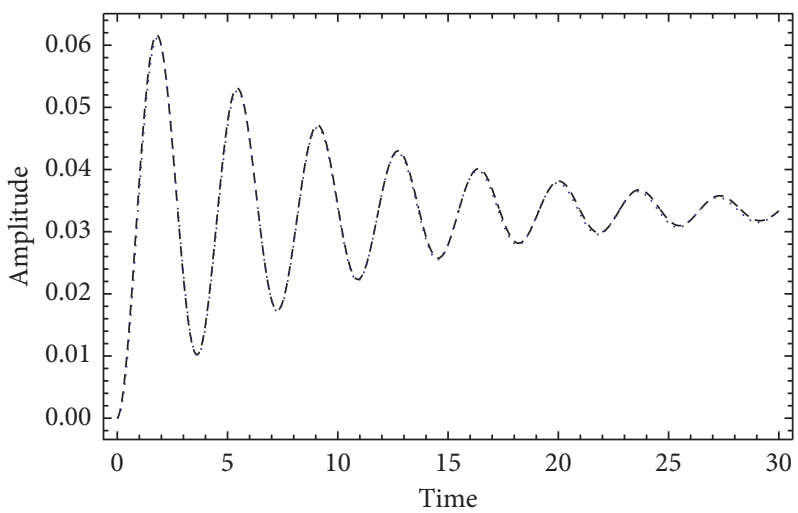

Figure 4: The comparison between the solution (43) and the RK4 numerical solution of the i.v.p. (40) is introduced for $\left(\gamma, \tilde{\alpha}, \tilde{\beta}, \delta, F, x_{0}, \dot{x}_{0}\right)=(0.1,3,0.5,0.1,0.1,0,0)$.

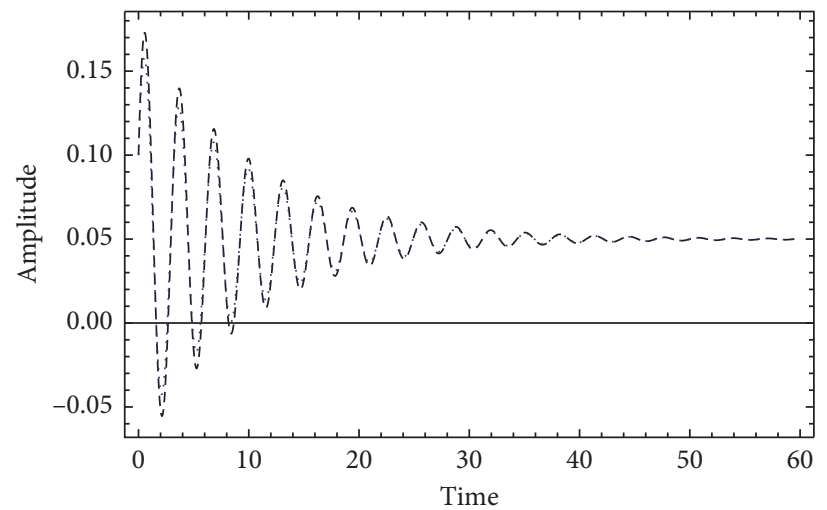

Figure 5: A comparison between the approximate analytical solution (50) (dotted curve) and the RK4 numerical solution (dashed curve) is investigated for RLC series circuits parameters $(R, L, C, E)$, i.e., for $\left(\alpha, \beta, \gamma, F, q_{0}, \dot{q}_{0}\right)=(4,1,0.1,0.2,0.1,0.2)$.

Figure 5 demonstrates the behavior of the damping oscillations in RLC series circuit for $\left(\alpha, \beta, \gamma, F, q_{0}, \dot{q}_{0}\right)=(4,1,0.1,0.2,0.1,0.2)$. Also, in this figure, we make a comparison between the approximate analytical solution (50) and the approximate numerical solution using the RK4, and the distance error $L_{D}(=0.0164089)$ is estimated. The harmony between two solutions has been observed, which confirms the high accuracy of the approximate analytical solutions.

\section{Conclusion}

Some novel solutions to the Duffing equation and its family (including the constant forced undamping Duffing equation and constant forced damping Duffing equation) have been derived in detail. First, we derived an analytical solution to the integrable constant forced undamping Duffing equation in the form of Weierstrass elliptic function with arbitrary initial conditions. Also, the periodicity of this solution has been obtained. Then, an approximate analytical (semianalytical) solution for the nonintegrable constant forced damping Duffing equation has been discussed depending on the exact solution of the unforced undamping (standard) Duffing equation. The relation between the constant forced damping Duffing equation and the constant forced damping pendulum equation, the constant forced damping cubic-quintic Duffing equation, and the constant forced damping Helmholtz-Duffing equation has been investigated. Some numerical examples have been introduced to demonstrate the obtained results and to make a comparison between the obtained solutions and RK4 numerical solutions. It was found from the results of the comparison that the semianalytical solution is compatible with the RK4 solution.

Future work: in some physical and engineering systems, the driving forces maybe a function of time and with an arbitrary circular frequency such as in the RLC circuits if the $\mathrm{AC}$ applied source. In this case, we obtain a new equation of motion which is called the damping Duffing equation with time-dependent driving force: $\left(\ddot{x}+2 \gamma \dot{x}+\alpha x+\beta x^{3}=F(t)\right)$. 
This equation of motion needs to be solved in the form of Weierstrass elliptic function, but it is outside the goal of the present work.

\section{Data Availability}

The data that support the findings of this study are available from the corresponding author upon request.

\section{Conflicts of Interest}

The authors declare that they have no conflicts of interest.

\section{Acknowledgments}

This study was supported by Taif University Researchers supporting project number (TURSP-2020/275), Taif University, Taif, Saudi Arabia.

\section{References}

[1] O. N. F. Nelson, Z. Yu, B. P. Dorian, and Y. Wang, "A new method for the exact solution of duffing equation," Journal of Applied Mathematics and Physics, vol. 6, no. 12, pp. 27182726, 2018.

[2] K. Johannessen, "The Duffing oscillator with damping," European Journal of Physics, vol. 36, p. 13, 2015 and references in therein, Article ID 065020.

[3] S. K. Lai and C. W. Lim, "Higher-order approximate solutions to a strongly nonlinear duffing oscillator," International Journal for Computational Methods in Engineering Science and Mechanics, vol. 7, no. 3, pp. 201-208, 2006.

[4] I. Kovacic and M. J. Brennan, The Duffing Equation: Nonlinear Oscillators and Their Behaviour, John Wiley \& Sons, Ltd., Hoboken, NJ, USA, 1st edition, 2011.

[5] P. S. Landa, Nonlinear Oscillations and Waves in Dynamical Systems, Springer, Berlin, Germany, 1996.

[6] G. Duffing, Erzwungene Schwingungen bei Veränderlicher Eigenfrequenz, F. Vieweg und Sohn, Braunschweig, Germany, 1918.

[7] J. Guckenheimer and P. J. Holmes, Nonlinear Oscillations, Dynamical Systems, and Bifurcations of Vector Fields, Springer Vrlag, Berlin, Germany, 1983.

[8] C. S. Hsu, "On the application of elliptic functions in nonlinear forced oscillations," Quarterly of Applied Mathematics, vol. 17, no. 4, pp. 393-407, 1960.

[9] E. T. Whittaker and G. N. Watson, A Course of Modern Analysis, Cambridge University Press, Cambridge, MA, USA, 4th edition, 1980.

[10] M. Abramowitz and I. Stegun, Handbook of Mathematical Functions, Dover, New York, NY, USA, 9th edition, 1980.

[11] A. H. Salas, E. Jairo, and H. Castillo, "Exact solutions to cubic duffing equation for a nonlinear electrical circuit," Journal of the American Mathematical Society, vol. 7, pp. 46-53, 2014.

[12] Ma'mon Abu Hammad, A. H. Salas, and S. A. El-Tantawy, "New method for solving strong conservative odd parity nonlinear oscillators: applications to plasma physics and rigid rotator," AIP Advances, vol. 10, p. 11, Article ID 085001, 2020.

[13] M. El-Shahed, "Application of differential transform method to non-linear oscillatory systems," Communications in Nonlinear Science and Numerical Simulation, vol. 13, no. 8, pp. 1714-1720, 2008.
[14] Y. Khan, M. Akbarzade, and A. Kargar, "Coupling of homotopy and the variational approach for a conservative oscillator with strong odd-nonlinearity," Scientia Iranica, vol. 19, no. 3, pp. 417-422, 2012.

[15] S. Nourazar and A. Mirzabeigy, "Approximated solution for nonlinear Duffing oscillator with damping effect using the modified differential transform method," Scientia Iranica, vol. 20, pp. 364-368, 2013.

[16] A. H. Salas and S. A. El-Tantawy, "On the approximate solutions to a damped harmonic oscillator with higher-order nonlinearities and its application to plasma physics: semianalytical solution and moving boundary method," The European Physical Journal Plus, vol. 135, pp. 833-917, 2020.

[17] K. Johannessen, "The Duffing oscillator with damping," The European Physical Journal Plus, vol. 36, p. 13, Article ID 065020, 2015.

[18] K. Johannessen, "The duffing oscillator with damping for a softening potential," International Journal of Applied and Computational Mathematics, vol. 3, no. 4, pp. 3805-3816, 2017.

[19] J.-w. Zhu, "A new exact solution of a damped quadratic nonlinear oscillator," Applied Mathematical Modelling, vol. 38, no. 24, pp. 5986-5993, 2014.

[20] A. E. Zúñga, "Application of jacobianan elliptic functions to the analysis of the steady-state solution of the damped duffing equation with driving force of elliptic type," Nonlinear Dynamics, vol. 42, pp. 175-184, 2005.

[21] U. R. Singh, G. Purohit, A. Sharma, and V. Patidar, "An analogue circuit to study the forced and quadratically damped Duffing oscillator," International Journal of Nonlinear Dynamics and Control, vol. 1, no. 1, pp. 87-96, 2017.

[22] U. R. Singh and G. Purohit, "Synchronization of quadratically damped mathieu-duffing based chaotic circuits with applications to secure communications," IJSR, vol. 5, pp. 80-85, 2016.

[23] W. D. Iwan, "On defining equivalent systems for certain ordinary non-linear differential equations," International Journal of Non-linear Mechanics, vol. 4, no. 4, pp. 325-334, 1969.

[24] B. Baumann, J. Schwieger, M. Wolff, F. Manders, and J. Suijker, "Nonlinear behavior in high-intensity discharge lamps," Journal of Physics D Applied Physics, vol. 49, p. 10, Article ID 255201, 2015. 